\title{
ST Elevation in a Patient With COVID-19 Infection-Associated Fever: A Case of Brugada Pattern
}

\author{
Guruprasad Mahadevaiah ${ }^{1}$, Abdul Aleem ${ }^{2}$, Antonio Secaira ${ }^{3}$, Samir Saba ${ }^{4}$, Nasir Shariff ${ }^{3}$ \\ 1. Pediatric Cardiology, California Northstate University College of Medicine, Sacramento, USA 2. Internal Medicine, \\ Lehigh Valley Hospital, Allentown, USA 3. Cardiology, Franciscan Heart and Vascular Associates, Tacoma, USA 4. \\ Cardiology, University of Pittsburgh Medical Center, Pittsburgh, USA
}

Corresponding author: Nasir Shariff, nasirshariff@gmail.com

\begin{abstract}
Coronavirus disease 2019 (COVID-19) is a global pandemic presenting with various cardiovascular manifestations. Although Brugada pattern ST-segment elevation (STE) is well described in patients admitted with febrile illness, the implication of recognizing this abnormality in patients with COVID-19 is critical in providing appropriate care for the patient and also reducing the exposure of healthcare professionals to the risk of infection. We report a patient with COVID-19 infection presenting with STE due to fever-related unmasking of Brugada pattern, who was managed conservatively.
\end{abstract}

Categories: Cardiology, Internal Medicine, Infectious Disease

Keywords: brugada pattern, st-segment elevation myocardial infarction (stemi), coronavirus infection, covid-19, electrocardiogram

\section{Introduction}

Percutaneous coronary intervention improves outcomes in patients with acute ST-segment elevation (STE) myocardial infarction [1]. Brugada pattern of STE is often misinterpreted as STE myocardial infarction resulting in inappropriate invasive studies. Prompt identification of the Brugada pattern of STE in patients with coronavirus disease 2019 (COVID-19) infection-associated fever could reduce invasive procedures and also the exposure of healthcare professionals to the risk of infection. We report a patient with COVID-19 infection presenting with STE due to fever-related unmasking of the Brugada pattern.

\section{Case Presentation}

A 40-year-old male with no prior significant medical history presented to the hospital with fever and chest pain of two-day duration. He described the chest pain as left sided and worse with lying down. There was no significant family history of sudden cardiac death or premature coronary artery disease. He denied history of tobacco use. Two of his family members had been tested positive for COVID-19 infection a week prior. On physical examination, his temperature was $39.1^{\circ} \mathrm{C}\left(102.3^{\circ} \mathrm{F}\right)$, blood pressure (BP) $131 / 88 \mathrm{mmHg}$, heart rate

Initial laboratory and radiologic investigation, which included complete blood count, comprehensive metabolic panel, and chest X-ray, was within normal limits. Initial troponin level was normal. D-Dimer was $224 \mathrm{ng} / \mathrm{mL}(0-243 \mathrm{ng} / \mathrm{mL})$.

Electrocardiogram (EKG) demonstrated a right bundle branch block morphology with a coved pattern of STE of $5 \mathrm{~mm}$ in the right precordial chest leads (Figure 1). The patient was initiated on heparin infusion, and cardiology was consulted. Considering the clinical history and EKG suggestive of Brugada pattern, a decision was made to hold off on any invasive procedures. The patient received antipyretics and was further evaluated with an echocardiogram. 


\section{Cureus}

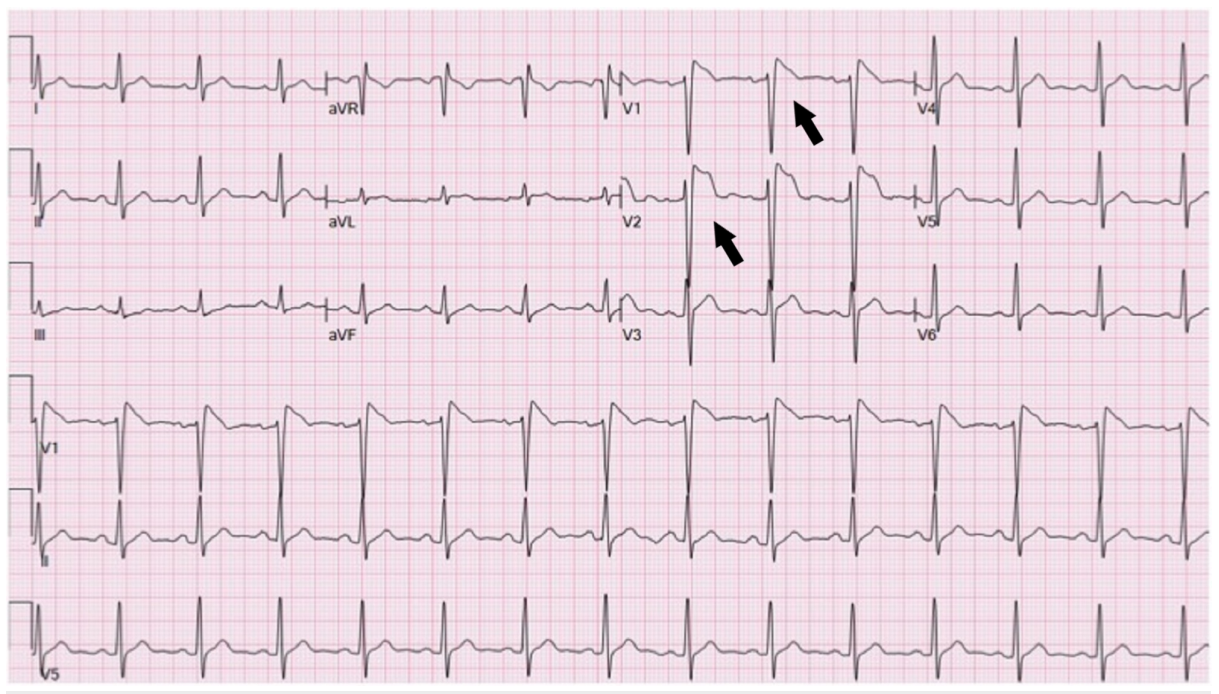

FIGURE 1: Electrocardiogram on initial presentation demonstrating Brugada type I pattern of ST-segment elevation in leads V1 and V2.

Echocardiogram demonstrated normal left ventricular (LV) function with no regional wall motion abnormality. Repeat EKG after 24 hours showed a significant resolution of the STE (Figure 2). Serial troponins were within normal limits. The patient was discharged the next day with information regarding isolation measures.

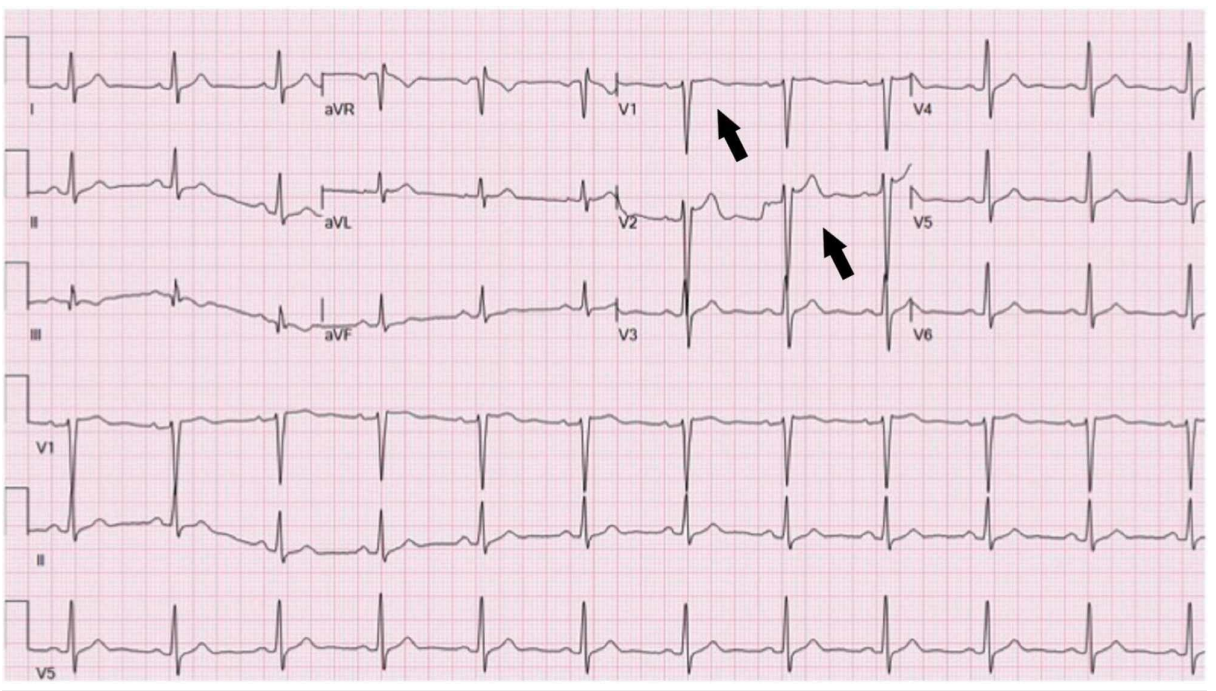

FIGURE 2: Electrocardiogram demonstrating significant resolution of ST-segment elevation following management of fever.

\section{Discussion}

Higher body temperatures result in reduced efficiency of the sodium channels presenting as Brugada pattern of STE in patients with a heterozygous mutation of sodium channels [2]. Patients with precipitated Brugada pattern without a history of syncope have a low risk of cardiac events [3]. Brugada pattern can be misinterpreted as STE myocardial infarction resulting in inappropriate invasive investigations. In patients presenting to the hospital with febrile illness, $2 \%$ were noted to have type I Brugada EKG pattern (coved pattern of STE of $>2 \mathrm{~mm}$ with symmetric negative T wave in the right precordial chest leads) [4]. Fever is the predominant clinical feature of patients admitted with COVID-19 [5]. In a case series study of patients with COVID-19 who had STE indicating potential acute myocardial infarction, nine of the 18 patients had undergone coronary angiogram procedure of which four patients were reported to have nonobstructive disease [6]. There have been two case reports of the Brugada pattern of EKGs in patients infected with COVID-19 [7,8]. The first published case report was a 49 -year-old male admitted after an episode of syncope and EKG showed Brugada pattern and a positive COVID-19 test [7]. The second published case report was a 
61-year-old male who presented with subjective fever, shortness of breath, chest pain, and noted reduced global LV function [8]. Due to underlying high-risk factors, patients in both reports underwent coronary angiogram procedure that demonstrated normal coronary anatomy.

Although coronary intervention significantly reduces the ischemia time and improves outcomes in patients with acute STE myocardial infarction, patients presenting with STE during the COVID-19 pandemic pose a challenging situation. Given the droplet transmission of COVID-19, invasive interventions are associated with a high risk of exposure of healthcare professionals to the virus. Also, with the positive pressure ventilation settings used in catheterization labs, there is a theoretical risk of further widespread contamination. Prompt identification of Brugada pattern in patients with febrile illness due to COVID-19 infection could enable timely appropriate care, reduce the risk of unnecessary invasive procedures, and protect healthcare professionals from the risk of exposure to the virus. Our case illustrates that fever due to COVID-19 infection can unmask Brugada pattern ST elevation, and patients can be managed with supportive care and antipyretics for the noted cardiac findings.

\section{Conclusions}

Our case illustrates that fever due to COVID-19 infection can unmask Brugada pattern ST elevation. Identification of the condition could result in timely appropriate care, reduce unnecessary invasive procedures, and protect healthcare professionals from the risk of infection.

\section{Additional Information \\ Disclosures}

Human subjects: Consent was obtained by all participants in this study. Conflicts of interest: In compliance with the ICMJE uniform disclosure form, all authors declare the following: Payment/services info: All authors have declared that no financial support was received from any organization for the submitted work. Financial relationships: All authors have declared that they have no financial relationships at present or within the previous three years with any organizations that might have an interest in the submitted work. Other relationships: All authors have declared that there are no other relationships or activities that could appear to have influenced the submitted work.

\section{References}

1. O'Gara PT, Kushner FG, Ascheim DD, et al.: 2013 ACCF/AHA guideline for the management of ST-elevation myocardial infarction: a report of the American College of Cardiology Foundation/American Heart Association Task Force on Practice Guidelines. Circulation. 2013, 127:362-425. 10.1161/CIR.0b013e3182742cf6

2. Keller DI, Rougier JS, Kucera JP, et al.: Brugada syndrome and fever: genetic and molecular characterization of patients carrying SCN5A mutations. Cardiovasc Res. 2005, 67:510-519. 10.1016/j.cardiores.2005.03.024

3. Priori SG, Napolitano C, Gasparini M, et al.: Natural history of Brugada syndrome: insights for risk stratification and management. Circulation. 2002, 105:1342-1347. 10.1161/hc1102.105288

4. Adler A, Topaz G, Heller K, et al.: Fever-induced Brugada pattern: how common is it and what does it mean? Heart Rhythm. 2013, 10:1375-1382. 10.1016/j.hrthm.2013.07.030

5. Cao Y, Liu X, Xiong L, Cai K: Imaging and clinical features of patients with 2019 novel coronavirus SARSCoV- 2: a systematic review and meta-analysis [Epub ahead of print]. J Med Virol. 2020, 10.1002/jmv.25822

6. Bangalore S, Sharma A, Slotwiner A, et al.: ST-segment elevation in patients with Covid-19: a case series . N Engl J Med. 2020, 382:2478-2480. 10.1056/NEJMc2009020

7. Chang D, Saleh M, Garcia-Bengo Y, Choi E, Epstein L, Willner J: COVID-19 infection unmasking Brugada syndrome. Heart Rhythm Case Rep. 2020, 6:237-240. 10.1016/j.hrcr.2020.03.012

8. Vidovich MI: Transient Brugada-like ECG pattern in a patient with coronavirus disease 2019 (COVID-19) [Epub ahead of print]. JACC Case Rep. 2020, 10.1016/j.jaccas.2020.04.007 